\title{
Growth mechanisms of ceria- and zirconia-based epitaxial thin films and hetero-structures grown by pulsed laser deposition
}

\author{
Daniele Pergolesi · Marco Fronzi · Emiliana Fabbri · \\ Antonello Tebano • Enrico Traversa
}

Received: 28 October 2012/Accepted: 7 December 2012/Published online: 22 December 2012

(C) The Author(s) 2013. This article is published with open access at Springerlink.com

\begin{abstract}
Thin films and epitaxial hetero-structures of doped and undoped $\mathrm{CeO}_{2}$, and $8 \mathrm{~mol} \% \mathrm{Y}_{2} \mathrm{O}_{3}$ stabilized $\mathrm{ZrO}_{2}$ (YSZ), were fabricated by pulsed laser deposition on different single crystal substrates. Reflection high energy electron diffraction was used to monitor in situ the growth mechanism of the films. Two distinct growth mechanisms were identified along the (001) growth direction for the Ce- and Zr-based materials, respectively. While the doped or undoped ceria films showed a 3-dimensional growth mechanism typically characterized by a pronounced surface roughness, YSZ films showed an almost ideal layerby-layer 2-dimensional growth. Moreover, when the two materials were stacked together in epitaxial hetero-structures, the two different growth mechanisms were preserved. As a result, a 2-dimensional reconstruction of the ceria-based layers determined by the YSZ film growing above was observed. The experimental results are explained in terms of the thermodynamic stability of the low-index surfaces of the two materials using computational analysis performed by density functional theory.
\end{abstract}

D. Pergolesi $(\bowtie) \cdot$ M. Fronzi · E. Fabbri

International Research Center for Materials Nanoarchitectonics (WPI-MANA), National Institute for Materials Science (NIMS), 1-1 Namiki, Tsukuba, Ibaraki 305-0044, Japan

e-mail: pergolesi.daniele@nims.go.jp

A. Tebano

CNR-SPIN and Dipartimento di Informatica Sistemi

e Produzione, University of Roma Tor Vergata, Rome, Italy

E. Traversa

International Research Center for Renewable Energy, State Key

Laboratory of Multiphase Flow in Power Engineering,

Xi' an Jiaotong University, Xi' an 710049, Shaanxi, China
Keywords Pulsed laser deposition · Hetero-structure . Oxygen-ion conducting oxides · Reflection high energy electron diffraction · Density functional theory

\section{Introduction}

Thin films of doped $\mathrm{CeO}_{2}$ and $\mathrm{Y}_{2} \mathrm{O}_{3}$-stabilized $\mathrm{ZrO}_{2}$ (YSZ) fabricated by different thin film deposition methods have been widely investigated as high temperature oxygen-ion conductors. Among the most important applications of these materials is the fabrication of electrolyte membranes for solid oxide fuel cells (SOFCs). Particularly, large oxygen ion conductivity characterizes doped $\mathrm{CeO}_{2}$. Typical dopants are $\mathrm{Gd}$ and $\mathrm{Sm}$ with concentration ranging from 10 to $20 \%$ [1]. The bulk oxygen-ion conductivity of $15 \% \mathrm{Sm}$-doped $\mathrm{CeO}_{2}$ (SDC) is as large as $0.02 \mathrm{~S} \mathrm{~cm}^{-1}$ at about $600{ }^{\circ} \mathrm{C}$, making this material one of the most performing solid state electrolyte in the so-called intermediate temperature range $\left(500-800{ }^{\circ} \mathrm{C}\right)$ [2]. YSZ is also widely used as an electrolyte, for SOFCs but mostly for oxygen sensors used to control the air-to-fuel ratio in vehicles, as well as for the fabrication of thermal barrier coatings due to its low thermal conductivity [3].

Pure zirconia $\left(\mathrm{ZrO}_{2}\right)$ shows a complicated phase diagram having a monoclinic crystal structure at temperatures below about $1,000{ }^{\circ} \mathrm{C}$, with transitions to tetragonal and cubic structures with increasing the temperature. Such phase transformations induce very large stresses that cause pure zirconia to crack, limiting its practical application. On the contrary, pure ceria $\left(\mathrm{CeO}_{2}\right)$ has a stable cubic phase that can easily become non-stoichiometric in oxygen content, showing important catalytic activity in oxygen reduction reaction processes. This material finds many practical applications, of which the most important are in 
catalysis [4]. Thin films of $\mathrm{CeO}_{2}$ have been also widely used as buffer layers for the growth of thin film heterostructures, such as insulating layers for microelectronic devices or as diffusion barriers to avoid chemical reactions at interfaces.

The increasing miniaturization of solid state electrochemical devices leads to an increasing importance of the thin film deposition technology for their fabrication. Besides, it is well known that the microstructural and morphological characteristics of thin films can strongly affect their physical and chemical properties. In particular, especially for doped ceria, the degree of crystallinity, the average grain size and the relative grain boundary extent can significantly modify the charge transport properties and the chemical stability in operating environment [5-7].

More recently, multilayered thin film hetero-structures comprising ceria-based oxides and/or YSZ have been fabricated in order to study the conducting properties of hetero-phase interfaces. A large increase in ionic conductivity was observed in case of incoherent interfaces due to the faster conduction pathways along dislocation lines $[8,9]$. More ordered oxygen-ion conducting hetero-interfaces have been also fabricated to investigate the effect on the conducting properties of the compressive or tensile strain occurring at quasi-coherent hetero-interfaces [10-12]. The fabrication of samples appropriately designed to allow isolating the effect of the interfacial strain field requires a careful control of the deposition process and a deep understanding of the growth mechanisms.

For this work, we used reflection high energy electron diffraction (RHEED) and X-ray diffraction (XRD) analyses to investigate the growth mechanism of doped and undoped ceria and YSZ thin films grown onto different single crystal substrates. Different growth mechanisms were observed for the two materials depending on the crystallographic growth direction.

Density functional theory calculations were used to theoretically investigate the thermodynamic stability of the (100) and (111) surfaces of the two materials in equilibrium with the gas phase, and a very good agreement with the experimental observations was found.

\section{Experimental}

Thin films of $\mathrm{CeO}_{2}, 15 \%$ Sm-doped $\mathrm{CeO}_{2}$ (SDC) and $8 \mathrm{~mol} \% \mathrm{Y}_{2} \mathrm{O}_{3}$ stabilized $\mathrm{ZrO}_{2}$ (YSZ) were grown by pulsed laser deposition (PLD) on different single crystal substrates listed in Table 1. Sintered ceramic pellets prepared in our laboratory were used as target materials.

The substrates were ultrasonically cleaned in de-ionized water, acetone and methanol, and dried with pure nitrogen prior to insertion into the deposition system. Before starting
Table 1 Single crystal substrates used in this work, their crystallographic structures, lattice parameters and crystallographic orientations

\begin{tabular}{llll}
\hline Material & Crystal structure & $\begin{array}{l}\text { Lattice } \\
\text { parameters } \\
(\mathrm{nm})\end{array}$ & $\begin{array}{l}\text { Crystal } \\
\text { orientation }\end{array}$ \\
\hline $\mathrm{MgO}$ & Cubic rock salt & $c=0.421$ & $(001)$ \\
$\mathrm{C}-$ cut $\mathrm{Al}_{2} \mathrm{O}_{3}$ & Hexagonal & $a=0.476$ & $(0001)$ \\
& & $b=0.476$ & \\
& & $c=1.300$ & \\
$9.5 \mathrm{~mol} \mathrm{YSZ}$ & Cubic fluorite & $c=0.512$ & $(001)$ \\
$\mathrm{YAlO}_{3}$ (YAO) & Orthorhombic & $a=0.518$ & $(110)$ \\
& perovskite & $b=0.531$ & \\
$\mathrm{SrTiO}_{3}$ (STO) & Cubic perovskite & $c=0.3905$ & $(001)$ \\
\hline
\end{tabular}

the deposition of the films, the substrates were kept at about $800{ }^{\circ} \mathrm{C}$ in about $40 \mathrm{~Pa}$ of high purity oxygen partial pressure for about $20 \mathrm{~min}$. It was observed that such a thermal treatment often resulted in an evident enhancement of the crystalline quality of the substrate surfaces.

The custom made PLD system (AOV Ltd) consisted of a vacuum chamber with a base pressure of about $10^{-6} \mathrm{~Pa}$, equipped with a load-lock chamber. The target carousel can hold up to six targets, and a stainless steel shield reduces cross contamination during the ablation process. Each target can simultaneously rotate and oscillate allowing a uniform ablation of the target surface. A KrF excimer laser (Coherent Lambda Physik $\mathrm{GmbH}$ ) with a wavelength of $248 \mathrm{~nm}$ and a pulse width of $25 \mathrm{~ns}$ was focused on the target material in a spot area of about $5 \mathrm{~mm}^{2}$. The energy of the laser shots onto the target surface was set at about $160 \mathrm{~mJ}$. A laser repetition rate between 2 and $5 \mathrm{~Hz}$ was used. The deposition of the films was carried out in an oxygen background pressure ranging from 0.5 to $5 \mathrm{~Pa}$, at a substrate temperature of about $700{ }^{\circ} \mathrm{C}$. The target-tosubstrate distance was $75 \mathrm{~mm}$.

The samples were cooled from the deposition temperature down to room temperature at 10 degrees $\mathrm{min}^{-1}$ in an oxygen background pressure of about $40 \mathrm{~Pa}$.

A high pressure reflection high energy electron diffraction (RHEED) system (AOV Ltd), equipped with a differential pumping system was used to monitor in situ the surface evolution of the films. An accelerating voltage of $28 \mathrm{kV}$ and an emission current of about $100 \mu \mathrm{A}$ were used. The RHEED patterns were recorded using a CCD camera.

$\mathrm{X}$-ray analysis (PANalytical X'pert Pro MPD) was used to calibrate the deposition rate by X-ray reflectometry (XRR) and to investigate the out-of-plane crystalline structure of the films by X-ray diffraction (XRD).

To confirm that the PLD process actually provided samples with the expected electrical properties, the 
electrical conductivities of the highly textured films of SDC and YSZ were measured by electrochemical impedance spectroscopy (EIS). Two Ti-Pt electrodes were deposited onto the film surfaces by electron beam deposition. The electrical characterization was performed in air, using a multichannel potentiostat VMP3 (Bio-Logic), in the frequency range between $1 \mathrm{MHz}$ and $100 \mathrm{mHz}$, varying the temperature between 400 and $700{ }^{\circ} \mathrm{C}$.

\section{Results and discussion}

The growth mechanism of ceria-based epitaxial thin films

Among the substrates used in this work, and listed in Table 1, (001)-oriented STO single crystals are particularly suitable for the epitaxial growth of doped and undoped $\mathrm{CeO}_{2}$ films [5]. $\mathrm{CeO}_{2}$ and SDC have a cubic fluorite crystal structure with a lattice parameter of about 5.41 and $5.44 \AA$, respectively. Epitaxial films can be obtained on STO with the in-plane orientation $(100) \mathrm{CeO}_{2} /(110) \mathrm{STO}$. Owing to this $45^{\circ}$ in-plane rotation of the $\mathrm{CeO}_{2}$ unit cell with respect to the STO unit cell, the resulting lattice misfit with the STO substrate is about $1.4 \%$ for SDC and about $2 \%$ for pure ceria. Figure 1a shows the $2 \theta-\theta$ scan of a $250 \AA$ A-thick film of SDC epitaxially oriented with the STO substrate. Figure $1 \mathrm{~b}$ shows the size effect interference fringes around the (002) SDC reflection, indicating the good crystallographic quality of the film. The red curve in Fig. 1b represents a simulation for a 45 unit cells thick SDC film (about $245 \AA$ ), which is in very good agreement with the expected thickness, as derived from the calibration of the deposition rate performed by XRR (Fig. 2b).

Figure 1c shows the typical RHEED patterns acquired for a film of SDC grown on STO, relative to the (100) in-plane orientation of the substrate. A spotty pattern associated with a 3D growth mechanism appeared after few laser shots implying that this island-like growth arose immediately at the early stage of the formation of the first layers.

Analogous results were obtained using (001)-oriented 9.5 YSZ single crystal substrates for the growth of epitaxial $\mathrm{CeO}_{2}$ films, as shown in Fig. 2. In this case, the two materials have the same crystalline structure and the $\mathrm{CeO}_{2}$ has a lattice misfit of about $-5.6 \%$ with respect to the substrate. Such a relatively large lattice misfit can be accommodated by the introduction of a regular network of misfit dislocations at the interface [13] allowing a wellordered cube-on-cube growth driven by the substrate along the $(001)$ direction. A mosaic spread of about $0.5^{\circ}$ was evaluated by measuring the FWHM of the Gaussian fit of the rocking curve acquired along the (002) reflection peak of the film.
Figure $2 b$ shows the XRR plot used for the calibration of the deposition rate of doped and undoped ceria. With the selected deposition parameters, a deposition rate of about $0.24 \AA$ shot $^{-1}$ was measured. Figure $2 \mathrm{c}$ shows that, also in this case, the RHEED patterns of the growing films showed the typical features of a $3 \mathrm{D}$ growth mechanism.

The spotty RHEED patterns characterizing the growth of pure and doped ceria films were observed over a relatively wide range of deposition parameters (substrate temperature of $700 \pm 100{ }^{\circ} \mathrm{C}$, oxygen background pressure from 0.1 up to $5 \mathrm{~Pa}$, deposition rate from 0.3 up to about $2 \AA \operatorname{shot}^{-1}$ ). Analogous RHEED patterns were also observed for (001)-oriented $\mathrm{CeO}_{2}$ films grown by means of different thin film deposition techniques [14].

To check whether such a 3D growth mechanism depends on the growth crystallographic axis, $\mathrm{C}$-cut $\mathrm{Al}_{2} \mathrm{O}_{3}$ (0001)oriented single crystalline substrates were used. $\mathrm{C}$-cut (0001) and R-cut (1102) sapphire crystals have been widely used for the growth of crystalline thin films of doped and undoped ceria. The R-cut surface is expected to favour the (001) orientation, while the C-cut should favour the (111)

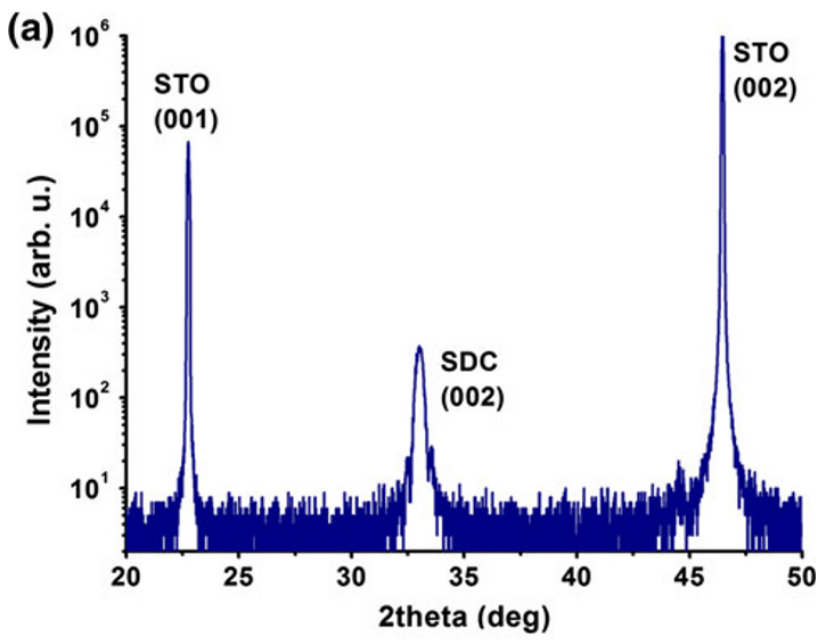

(b)
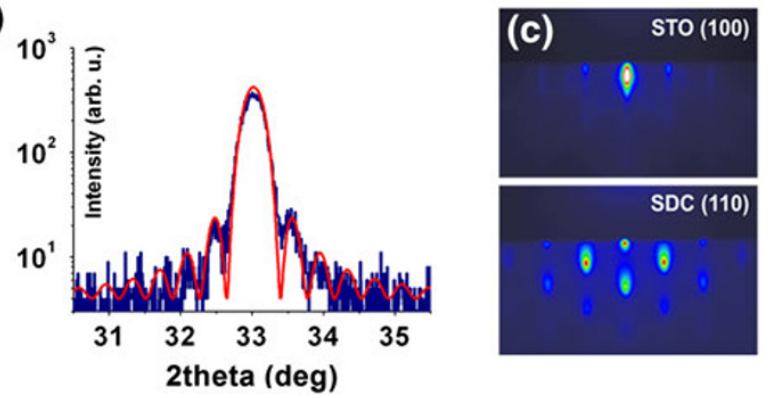

Fig. 1 a XRD analysis of an SDC film grown on STO. b XRD plot of the (002) SDC reflection with superimposed simulation (red curve) of the size effect interference fringes for an SDC film thickness of 45 unit cells. c RHEED pattern recorded along the in-plane (100) orientation of the substrate and RHEED pattern of the SDC film at the end of the deposition 


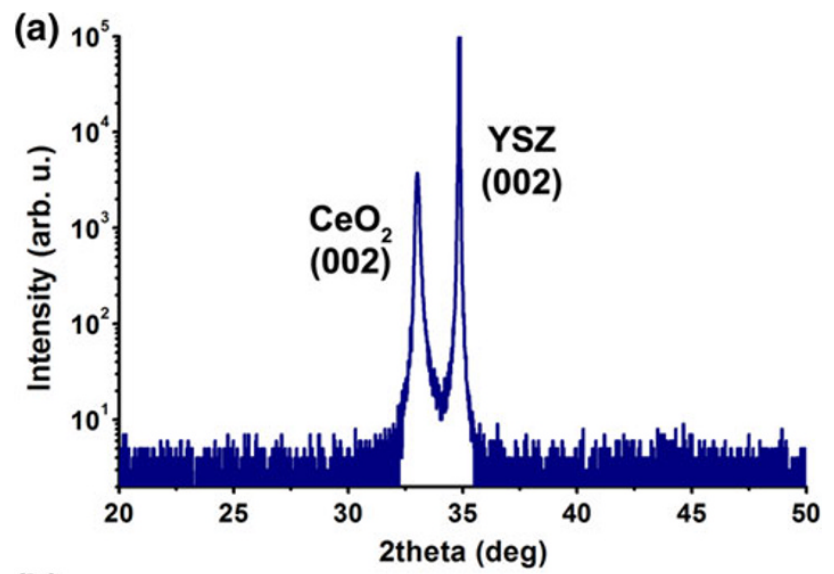

(b)

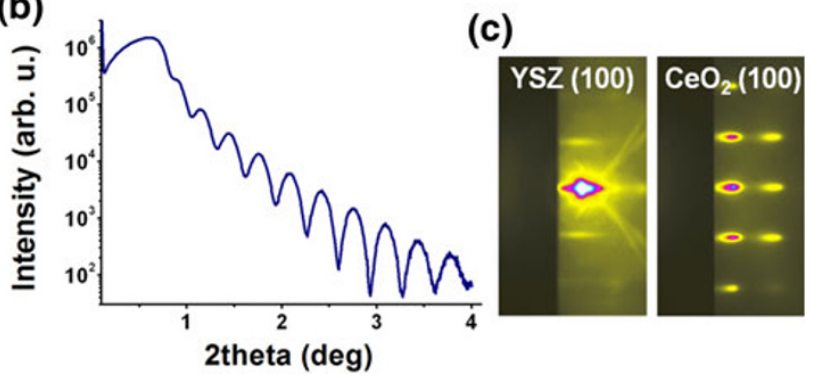

Fig. 2 a XRD analysis of a $\mathrm{CeO}_{2}$ film grown on a (001)-oriented 9.5 YSZ substrate. $\mathbf{b}$ XRR measurement performed for the calibration of the deposition rate of the film. c RHEED patterns of the substrate and of the film recorded along the in-plane (100) orientation of the substrate

film orientation $[15,16]$. Nevertheless, both orientations were obtained on both surfaces, as well as films showing mixed $(001) /(111)$ orientation, depending on process parameters and deposition technique $[17,18]$. Using PLD, the fabrication of highly (111)-oriented $\mathrm{CeO}_{2}$ films on C-cut sapphire has been reported for example in [6].

Figure 3 shows that preferentially (111)-oriented SDC films were grown with a minor (001) orientation. We observed that, in our experimental condition, (111)-oriented films were obtained at relatively low oxygen partial pressure (in the order of 0.1-0.5 Pa), while for larger values of oxygen partial pressure (few Pa), the films showed well-defined mixed (001)/(111) orientation.

The RHEED patterns acquired during the growth at low oxygen partial pressure (inset in Fig. 3) showed the typical features that characterize a quasi-2D layer-by-layer growth mechanism. Such streaky features were never observed for $\mathrm{CeO}_{2}$ or SDC film grown onto STO or YSZ substrates where the growth was along the (001) axis.

To summarize, these measurements showed that doped or undoped ceria presents two different growth mechanisms depending on the growth directions; the film grows predominantly quasi-2D along the (111) direction, while an evident 3D growth mechanism was observed along the (001) direction.

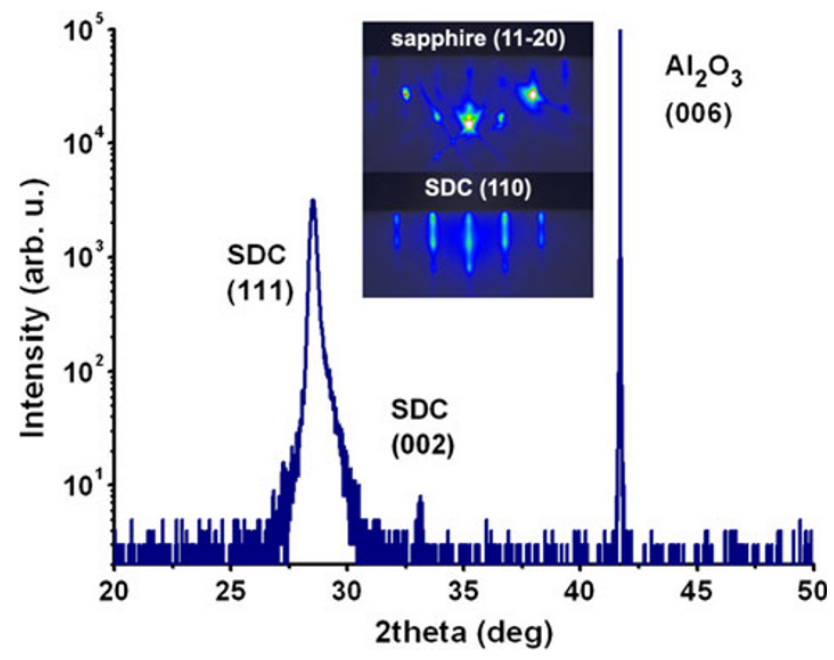

Fig. $3 \mathrm{XRD}$ analysis of an SDC film grown on $\mathrm{C}$-cut $\mathrm{Al}_{2} \mathrm{O}_{3}$ single crystal substrate. The inset shows the RHEED patterns recorded for the substrate and for the SDC film at the end of the deposition

Finally, the electrical conductivity of an SDC film grown on sapphire was measured by EIS in air. The measured conductivity ranged from $0.03 \mathrm{~S} \mathrm{~cm}^{-1}$ at about $680{ }^{\circ} \mathrm{C}$ down to $0.001 \mathrm{~S} \mathrm{~cm}^{-1}$ at about $400{ }^{\circ} \mathrm{C}$, with activation energy of about $0.70 \mathrm{eV}$. This result is in very good agreement with the literature data relative to the bulk conductivity of doped $\mathrm{CeO}_{2}$ films [6]. The electrical characterization of the films grown on STO and YSZ single crystal wafers cannot give reliable results due to the conductive properties of the deposition substrates at high temperatures.

The growth mechanism of YSZ epitaxial thin films

The growth of YSZ films was studied using (110)-oriented YAO and (001)-oriented $\mathrm{MgO}$ single crystal substrates. YSZ has a cubic fluorite structure with lattice parameter of about $5.14 \AA$, which results in a lattice misfit of about $1.5 \%$ with (110)-oriented YAO (Table 1). Figure 4a shows the out-of-plane XRD analysis of a YSZ film grown on YAO. The presence of well-defined size effect interference fringes around the (002) reflection line of the film (Fig. 4b) suggests a very good crystallographic quality. The black curve in Fig. 4b shows a simulation for a 35 unit cells thick YSZ film (about $180 \AA$ ). From this simulation, we could estimate a deposition rate of about $0.059 \AA \operatorname{shot}^{-1}$.

Opposite to what observed in the case of (001)-oriented ceria-based films, the RHEED patterns of (001)-oriented YSZ films clearly showed a different growth mechanism. Figure $3 \mathrm{~b}$ shows the RHEED patterns of the substrate and of the YSZ film acquired toward the (100) in-plane direction of YAO. The RHEED patterns consisted of 

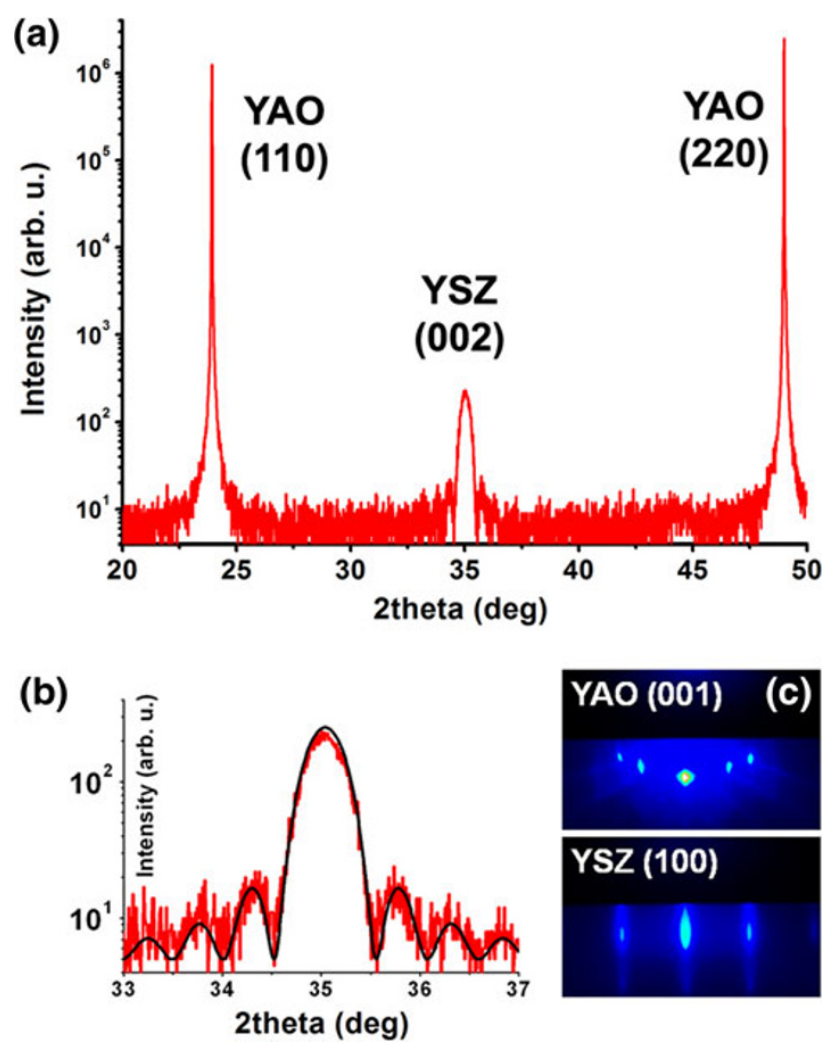

Fig. 4 a XRD analysis of a YSZ film grown on (110)-oriented YAO. b XRD plot of the (002) YSZ reflection with superimposed simulation (black curve) of the size effect interference fringes for a YSZ film thickness of 35 unit cells. c RHEED patterns of the substrate and the YSZ film recorded along the in-plane (100) orientation of the substrate

well-defined streaks revealing a 2D layer-by-layer growth mechanism.

Very similar result was obtained analysing a $300 \AA$ thick film of YSZ grown on (001)-oriented $\mathrm{MgO}$ substrate. In spite of a lattice misfit as large as $-18 \%$, a highly textured growth was observed by XRD analysis (Fig. 5). A relatively large value of $0.64^{\circ}$ was found for the FWHM of the (002) $\omega$-scan of the film. Figure 5a shows the XRR plot used to measure the deposition rate of the film with the selected deposition parameters. A value of about $0.053 \AA$ shot $^{-1}$ was estimated in very good agreement with the value obtained from the simulation of the size effect interference fringes showed in Fig. 4b. The final RHEED pattern showed the typical features associated with a 2D layer-bylayer growth (Fig. 5b). However, in this case, the RHEED pattern disappeared during the deposition of the first layers, suggesting the formation of a disordered interface probably characterized by a large density of misfit dislocations introduced to release the excess interfacial strain [8].

According to the measured deposition rate, a streaky RHEED pattern originated from the surface of the YSZ film growing on $\mathrm{MgO}$ was not observed below a minimum

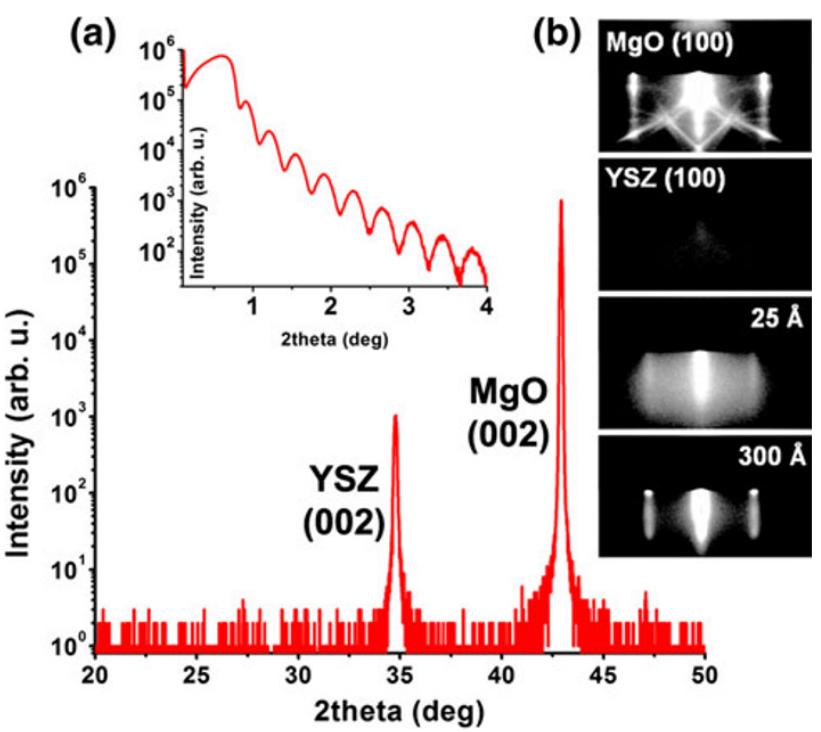

Fig. 5 a XRD analysis of a YSZ film grown on (001)-oriented MgO substrate. The inset shows the XRR measurement performed for the calibration of the deposition rate of the film. $\mathbf{b}$ RHEED patterns of the substrate and of the film recorded along the in-plane (100) orientation of the substrate

thickness of about $20 \AA$. However, even in the case of a particularly unfavourable crystalline matching with the deposition substrate, YSZ showed a clear tendency to a 2D growth toward the (001) direction driven by the deposition substrate.

The electrical conductivity of a YSZ film grown on $\mathrm{MgO}$ was measured in air by EIS and the conductivity was found to range from $9.6 \times 10^{-3}$ down to $8.5 \times 10^{-5} \mathrm{~S} \mathrm{~cm}^{-1}$ at 700 and $400{ }^{\circ} \mathrm{C}$, respectively, showing an activation energy of about $0.98 \mathrm{eV}$. The measured conductivity was in excellent agreement with the conductivity of YSZ single crystals [19].

\section{Calculation of the low index surface energies}

The comparison between the results obtained with YSZ and those obtained with doped and undoped ceria films strongly suggests a significant difference in the thermodynamic stability of the (001) surfaces of the two materials. To understand the driving mechanism for the different behaviours experimentally observed, a theoretical evaluation of the surface energy of the low-index surface orientation of $\mathrm{CeO}_{2}$ and cubic zirconia $\left(\mathrm{c}-\mathrm{ZrO}_{2}\right)$ was computed using first-principles density functional theory (DFT).

The low-index surface energies of $\mathrm{CeO}_{2}$ nano-particles have been analysed in a previous work and the stable surfaces were identified as a function of the temperature, the chemical potential and the oxygen background pressure [20]. It was found that unless the chemical potential of the oxygen is extremely low (i.e. very high temperature and very 
low oxygen partial pressure), the (111) surface orientation is stable if compared with the (100) and (110). By minimization of the Gibbs free energy as indicated by the GibbsWulff theorem, it was possible then to calculate the equilibrium crystal shape of a $\mathrm{CeO}_{2}$ nano-particles and show that under oxygen-rich conditions, the (111) surface is the only orientation present in the particle and the resulting morphology is described as the octahedron shown in Fig. 6a.

For this work, an analogous analysis has been carried out to calculate the low-index surface energies of $\mathrm{c}-\mathrm{ZrO}_{2}$ nano-particles. As previously mentioned, the cubic fluorite zirconia is stable only at very high temperatures. In YSZ, the effect of the dopant $\left(\mathrm{Y}_{2} \mathrm{O}_{3}\right)$, besides creating oxygen vacancies allowing oxygen-ion diffusion, is to stabilize the cubic structure at lower temperatures.

Although YSZ is the material under investigation in this work, for the calculation of the low-index surface energies, we only considered the cubic undoped crystal, since it has been shown that the trend of the low-index surface relative stability for c- $\mathrm{ZrO}_{2}$ and YSZ does not change [21]. Therefore, the computed surface energies obtained considering undoped cubic zirconia are expected to bring, at least qualitatively, to the same conclusions.

The surface energies of (111) and (001) surfaces of $\mathrm{c}-\mathrm{ZrO}_{2}$ nano-particles under oxygen-rich condition were calculated. Table 2 compares the low-index surface energies calculated for $\mathrm{CeO}_{2}$ and $\mathrm{c}-\mathrm{ZrO}_{2}$ nano-particles, showing that both crystalline structures favour the (111) surface that strongly minimize the Gibbs free energy, but the difference between the (001) and (111) surface energies in the case of $\mathrm{c}-\mathrm{ZrO}_{2}$ turned out to be about a factor of two smaller than in the case of $\mathrm{CeO}_{2}$.

The equilibrium crystal shape of c- $\mathrm{ZrO}_{2}$ nano-particles, calculated according to the Wulff construction by minimizing the surface energy, can be described as a truncated octahedron showing a mixture of (001) and (111) surfaces (Fig. 6b).

The Wulff theorem states that for a given volume, the crystal exposes different surface orientations in order to
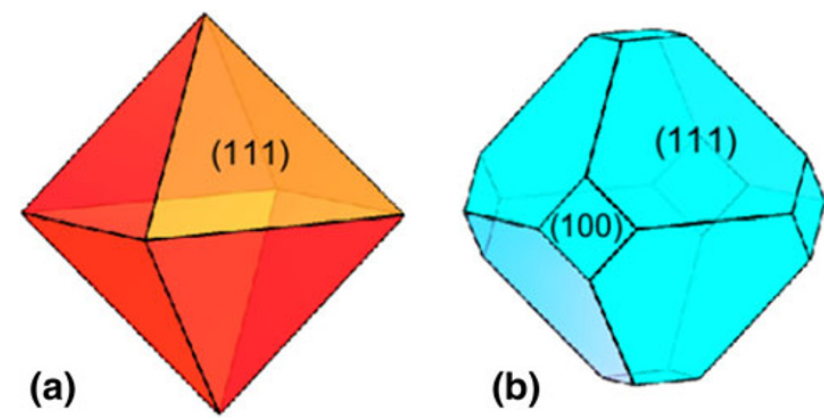

Fig. 6 Morphology of the equilibrium crystal shape nano-particles of $\mathrm{CeO}_{2}$ (a) and $\mathrm{c}-\mathrm{ZrO}_{2}$ (b) obtained by the minimization of the Gibbs free energy, as indicated by the Gibbs-Wulff theorem
Table 2 Low index surface Gibbs free energy of ceria and cubic zirconia

\begin{tabular}{lllll}
\hline Material & $\begin{array}{l}\text { Surface } \\
\text { index }\end{array}$ & $\begin{array}{l}\text { Surface Gibbs } \\
\text { free energy }(\mathrm{eV})\end{array}$ & $\begin{array}{l}\text { Surface energy } \\
\text { difference }(\mathrm{eV})\end{array}$ & References \\
\hline $\mathrm{CeO}_{2}$ & $(001)$ & 0.230 & 0.193 & {$[20]$} \\
& $(111)$ & 0.037 & & This work \\
$\mathrm{c}-\mathrm{ZrO}_{2}$ & $(001)$ & 0.189 & 0.116 & \\
& $(111)$ & 0.073 & & \\
\hline
\end{tabular}

minimize the surface energy. In the case of highly textured thin films on single crystal substrates, the epitaxial stress can modify the extension of the different facets present in the stress-free equilibrium shape, but it cannot create new facets [22]. In this sense, we can say that the analysis proposed here for nano-particles may give an indication of the local preferential surface orientation of a thin film at thermodynamic equilibrium.

In both cases $\left(\mathrm{CeO}_{2}\right.$ and $\left.\mathrm{c}-\mathrm{ZrO}_{2}\right)$, the (111) surface is stable if compared with other low-index orientations. Even though the growth is driven toward the (001) direction by crystalline constrains, the (111) facet is strongly favoured for $\mathrm{CeO}_{2}$, which will arrange the lattice in order to expose the (111) surface. As a result, the favoured (001) surface will consist on a mosaic of (111)-facet octahedra (001)oriented, thus enhancing the surface roughness. On the other hand, $\mathrm{c}-\mathrm{ZrO}_{2}$, having a smaller difference between the (001) and (111) surface energies will expose a mixture of these two orientations originating smoother surfaces.

The growth mechanism of ceria- and zirconia-based superlattices

Multilayered hetero-structures and superlattices made by coupling SDC or YSZ with an insulating phase $[9,11,12]$ have been fabricated to study the conducting properties of the interfaces [23]. To the best of our knowledge, for these hetero-structures, high crystalline quality (biaxial texture, no grain boundary) has been achieved only for (001)oriented YSZ-STO multilayers grown by PLD on STO substrates [24], and for (001)-oriented $\mathrm{CeO}_{2}-\mathrm{YSZ}$ heterostructures grown on $\mathrm{MgO}[25,26]$.

Highly (111)-oriented hetero-structures of Gd-doped ceria and Gd-doped zirconia, with minor phases from other orientations, were obtained on C-cut sapphire by molecular beam epitaxy [10]. In this case, the authors reported the observation of a streaky RHEED pattern up to a film thickness of about $40 \mathrm{~nm}$ with additional weak features corresponding to polycrystalline structure with increasing the thickness of the deposition.

The literature does not report any detailed study of the growth mechanism of these hetero-structures, particularly in the case of the (001) orientation. 


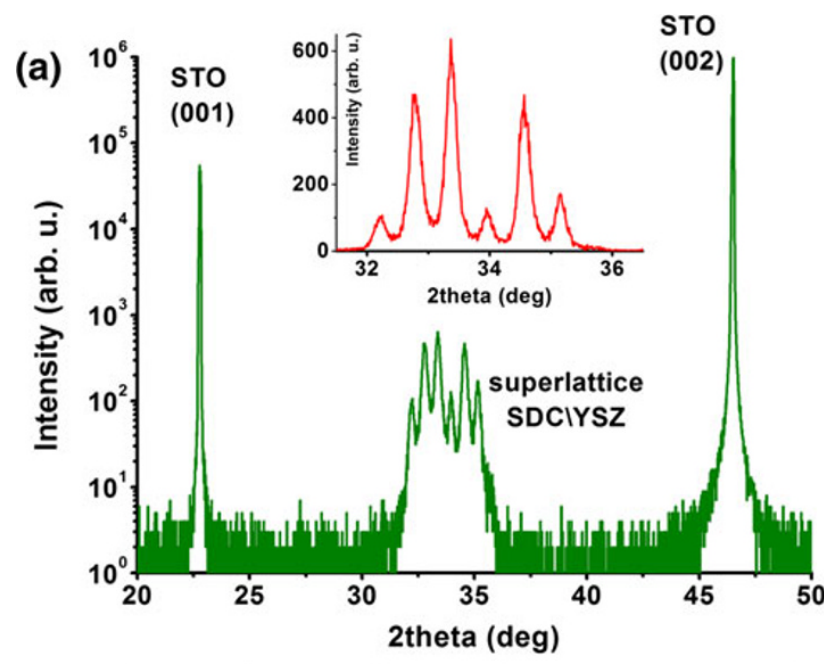

(b)
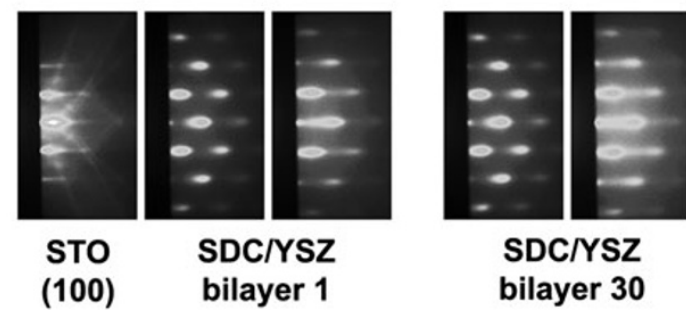

Fig. 7 a XRD analysis of an SDC/YSZ superlattice grown on (001)oriented STO substrate. b RHEED patterns acquired during the growth of the superlattice along the (100) in-plane orientation of the STO substrate

For this work, we fabricated superlattices made of SDC and YSZ on (001)-oriented STO substrates and superlattices made of $\mathrm{CeO}_{2}$ and $\mathrm{YSZ}$ on (001)-oriented $\mathrm{MgO}$ substrates. The growth of each layer was monitored by RHEED.

The SDC/YSZ superlattice consisted of 30 bilayers of the two materials with single layer thickness of about 30 unit cells, as estimated from the deposition rate measured by XRR. Figure $7 \mathrm{a}$ shows the XRD analysis of such a superlattice. The superlattice was epitaxially (001)-oriented with the STO substrate and the XRD plot showed the typical superlattice peaks, as shown in the inset in Fig. 7a.

The RHEED patterns of several SDC/YSZ bilayers were recorded during the growth of the superlattice. Figure $7 \mathrm{~b}$ shows the electron reflection patterns relative to the first and the 30th final bilayers. RHEED diagnostic revealed that the two materials, SDC and YSZ, showed the two different growth mechanisms described above along this crystallographic orientation, i.e. a 3D growth for SDC and a quasi-2D growth for YSZ.

Figure 8 shows the XRD plot (Fig. 8a) and the RHEED patterns (Fig. 8 b) of the $\mathrm{CeO}_{2} / \mathrm{YSZ}$ superlattice that consisted of $15 \mathrm{CeO}_{2} / \mathrm{YSZ}$ bilayers grown on $\mathrm{MgO}$ using a thin buffer layer of STO, about $5 \mathrm{~nm}$ thick. According to the XRR measurement of the deposition rate, the expected

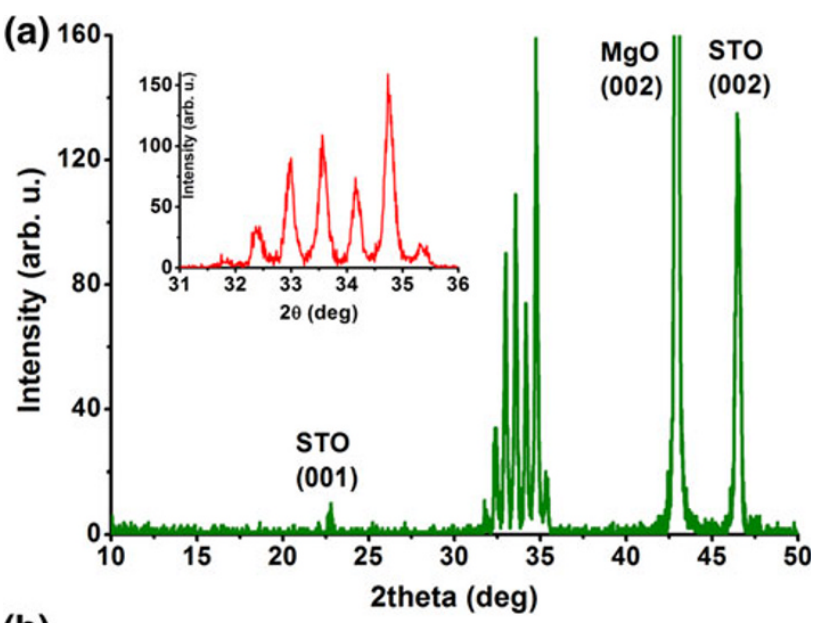

(b)
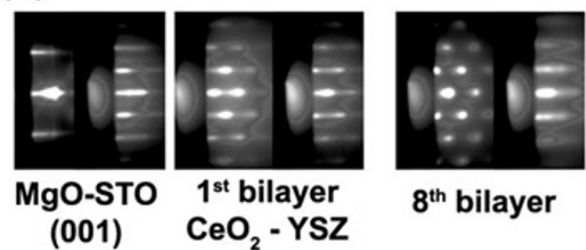

$8^{\text {th }}$ bilayer

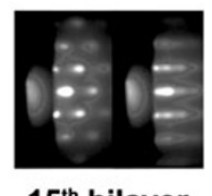

$15^{\text {th }}$ bilayer

Fig. 8 a XRD analysis of a $\mathrm{CeO}_{2} / \mathrm{YSZ}$ superlattice grown on (001)oriented $\mathrm{MgO}$ substrate using a $5 \mathrm{~nm}$ thick STO buffer layer. b RHEED patterns acquired during the growth of the superlattice along the (100) in-plane orientation of the $\mathrm{MgO}$ substrate

thickness of each individual layer was about 15 unit cells. The very well-defined superlattice features allowed identifying the diffraction satellite peaks up to the third order. For the fabrication of this sample, $\mathrm{MgO}$ was selected as deposition substrate due to its good insulating properties at high temperatures that allow the electrical characterization along the planar direction of the hetero-interfaces. The STO buffer layer provides the suitable lattice matching for the epitaxial growth [5].

An almost ideal 2D layer-by-layer growth with cube-oncube symmetry was observed for the STO buffer layer on $\mathrm{MgO}$. Also in this case, a regular array of spots characterized the RHEED patterns of all the $\mathrm{CeO}_{2}$ layers, while streaky patterns were observed for all the YSZ layers.

Both Fig. 7 (SDC-YSZ on STO) and Fig. $8\left(\mathrm{CeO}_{2}-\mathrm{YSZ}\right.$ on STO-buffered $\mathrm{MgO}$ ) show a mechanism of 2D surface reconstruction of the ceria layers determined by the YSZ layer growing above. A similar mechanism of roughness suppression induced by the deposition of a second layer was already observed during the growth of superlattice structures [27].

\section{Conclusions}

Thin films of $\mathrm{CeO}_{2}, 15 \%$ Sm-doped $\mathrm{CeO}_{2}$ (SDC), as well as $8 \mathrm{~mol} \%$ yttria-doped zirconia (YSZ) were grown on different single crystalline substrates. RHEED diagnostic 
allowed identifying two distinct growth mechanisms for the ceria films along the (001) and (111) growth directions. A 3D growth mechanism, associated with a more pronounced surface roughness, clearly characterized the growth of the (001)-oriented surfaces, while a quasi-2D growth was observed for the (111)-oriented surfaces. The 3D (001)-oriented growth of $\mathrm{CeO}_{2}$ and SDC was observed over a relatively wide range of deposition temperatures, laser energy densities, oxygen background pressures, and deposition rates, suggesting that this growth mechanism is intrinsically related with the physicochemical properties of this material. On the contrary, YSZ films showed an almost ideal 2D layer-by-layer growth along the (001) orientation, even in the presence of relatively unfavourable crystalline matching with the substrate, such as in the case of films grown on $\mathrm{MgO}$.

The two distinct growth mechanisms along the (001) orientation were, to the best of our knowledge, for the first time correlated with the low index surface Gibbs free energies of the two materials, computed by first-principles DFT. Our calculation showed that both crystalline structures favour the (111) surface that strongly minimizes the Gibbs free energy, but the difference between the (001) and (111) surface energies of $\mathrm{CeO}_{2}$ is twice that of cubic $\mathrm{ZrO}_{2}$.

The enhanced roughness of the (001)-oriented doped or undoped $\mathrm{CeO}_{2}$ surfaces should be taken into account in particular as far as the growth of highly textured multilayered hetero-structures is concerned. In this work, we have shown that the ceria-based layers preserve their characteristic 3D growth mechanism also when comprised in super-structures with YSZ leading to potentially significant effects on the interface morphology. Therefore, especially for $\mathrm{CeO}_{2}$ and SDC, much better surface (and interface) quality might be achieved by developing the expertise needed to obtain coherent film epitaxy and biaxial texture along less common crystallographic directions, for instance on pseudo-cubic (110)- or (111)-oriented surfaces.

Acknowledgments This work was partly supported by the World Premier International Research Centre Initiative of MEXT, Japan.

Open Access This article is distributed under the terms of the Creative Commons Attribution License which permits any use, distribution, and reproduction in any medium, provided the original author(s) and the source are credited.

\section{References}

1. Esposito, V., Traversa, E.: Design of electroceramics for solid oxides fuel cell applications: playing with ceria. J. Am. Ceram. Soc. 91, 1037-1051 (2008)

2. Wachsman, E.D., Lee, K.Y.: Lowering the temperature of solid oxide fuel cells. Science 334, 935-939 (2011)
3. Chen, L.B.: Yttria-stabilized zirconia thermal barrier coatings-a review. Surf. Rev. Lett. 13, 535-544 (2006)

4. Trovarelli, A.: Catalysis by ceria and related materials. In: Catalytic science series, vol. 2. World Scientific Publishing Co, Singapore (2002)

5. Sanna, S., Esposito, V., Pergolesi, D., Orsini, A., Tebano, A., Licoccia, S., Balestrino, G., Traversa, E.: Fabrication and electrochemical properties of epitaxial samarium-doped ceria films on $\mathrm{SrTiO}_{3}$-buffered $\mathrm{MgO}$ substrates. Adv. Funct. Mater. 19, 1713-1719 (2009)

6. Goebel, M.C., Gregori, G., Guo, X., Maier, J.: Boundary effects on the electrical conductivity of pure and doped cerium oxide thin films. Phys. Chem. Chem. Phys. 12, 14351-14361 (2010)

7. Goebel, M.C., Gregori, G., Maier, J.: Mixed conductivity in nanocrystalline highly acceptor doped cerium oxide thin films under oxidizing conditions. Phys. Chem. Chem. Phys. 13, 1094010945 (2011)

8. Sillassen, M., Eklund, P., Pryds, N., Johnson, E., Helmersson, U., Bøttiger, J.: Low-temperature superionic conductivity in strained yttria-stabilized zirconia. Adv. Funct. Mater. 20, 2071-2076 (2010)

9. Korte, C., Peters, A., Janek, J., Hesse, D., Zakharov, N.: Ionic conductivity and activation energy for oxygen ion transport in superlattices-the semicoherent multilayer system YSZ $\left(\mathrm{ZrO}_{2}+9.5 \mathrm{~mol} \% \mathrm{Y}_{2} \mathrm{O}_{3}\right) / \mathrm{Y}_{2} \mathrm{O}_{3}$. Phys. Chem. Chem. Phys. 10, 4623-4635 (2008)

10. Azad, S., Marina, O.A., Wang, C.M., Saraf, L., Shutthanandan, V., McCready, D.E., El-Azab, A., Jaffe, J.E., Engelhard, M.H., Peden, C.H.F., Thevuthasan, S.: Nanoscale effects on ion conductance of layer-by-layer structures of gadolinia-doped ceria and zirconia. Appl. Phys. Lett. 86, 1319061 (2005)

11. Korte, C., Schichtel, N., Hesse, D., Janek, J.: Influence of interface structure on mass transport in phase boundaries between different ionic materials. Monatsh. Chem. 140, 1069-1080 (2009)

12. Schichtel, N., Korte, C., Hesse, D., Zakharov, N., Butz, B., Gerthsenc, D., Janek, J.: On the influence of strain on ion transport: microstructure and ionic conductivity of nanoscale YSZ/Sc2O3 multilayers. Phys. Chem. Chem. Phys. 12, 1459614608 (2010)

13. Wang, C.M., Thevuthasan, S., Peden, C.H.F.: Interface structure of an epitaxial cubic ceria film on cubic zirconia. J. Am. Ceram. Soc. 86, 363-365 (2003)

14. Ikegawa, S., Motoi, Y.: Growth of $\mathrm{CeO}_{2}$ thin films by metalorganic molecular beam epitaxy. Thin Solid Films 281, 60-63 (1996)

15. Zaitsev, A.G., Ockenfuss, G., Guggi, D., Wördenweber, R., Krüger, U.: Structural perfection of (001) $\mathrm{CeO}_{2}$ thin films on (1102) sapphire. J. Appl. Phys. 81, 3069-3072 (1997)

16. Bera, D., Kuchibhatla, S.V.N.T., Azad, S., Saraf, L., Wang, C.M., Shutthanandan, V., Nachimuthu, P., McCready, D.E., Engelhard, M.H., Marina, O.A., Baer, D.R., Seal, S., Thevuthasan, S.: Growth and characterization of highly oriented gadolinia-doped ceria (111) thin films on zirconia (111)/sapphire (0001) substrates. Thin Solid Films 516, 6088-6094 (2008)

17. Kurian, J., Naito, M.: Growth of epitaxial $\mathrm{CeO}_{2}$ thin films on r-cut sapphire by molecular beam epitaxy. Physica C 492, 31-37 (2004)

18. Nandasiri, M.I., Nachimuthu, P., Varga, T., Shutthanandan, V., Jiang, W., Kuchibhatla, S.V.N.T., Thevuthasan, S., Seal, S., Kayani, A.N.: Influence of growth rate on the epitaxial orientation and crystalline quality of $\mathrm{CeO}_{2}$ thin films grown on $\mathrm{Al} 2 \mathrm{O} 3$ (0001). J. Appl. Phys. 109, 013525 (2011)

19. Manning, P.S., Sirman, J.D., De Souza, R.A., Kilner, J.A.: The kinetics of oxygen transport in $9.5 \mathrm{~mol} \%$ yttria stabilized zirconia. Solid State Ionics 100, 1-10 (1997) 
20. Fronzi, M., Soon, A., Delley, B., Traversa, E., Stampfl, C.: Stability and morphology of cerium oxide surfaces in an oxidizing environment: a first-principles investigation. J. Chem. Phys. 131, 104701 (2009)

21. Xia, X., Oldman, R., Catlow, R.: Computational modeling study of bulk and surface of yttria-stabilized cubic zirconia. Chem. Mater. 21, 3576-3585 (2009)

22. Muller, P., Kern, R.: Equilibrium nano-shape change induced by epitaxial stress: effect of surface stress. Surf. Sci. 457, 229-253 (2000)

23. Fabbri, E., Pergolesi, D., Traversa, E.: Ionic conductivity in oxide heterostructures: the role of interfaces. Sci. Technol. Adv. Mater. 11, 054503-054512 (2010)

24. Garcia-Barriocanal, J., Rivera-Calzada, A., Varela, M., Sefrioui, Z., Iborra, E., Leon, C., Pennycook, S.J., Santamaria, J.: Colossal ionic conductivity at interfaces of epitaxial $\mathrm{ZrO}_{2}: \mathrm{Y}_{2} \mathrm{O}_{3} / \mathrm{SrTiO}_{3}$ heterostructures. Science 321, 676-680 (2008)

25. Pergolesi, D., Tebano, A., Fabbri, E., Balestrino, G., Licoccia, S., Traversa, E.: Pulsed lased deposition of superlattices based on ceria and zirconia. ECS Trans. 35, 1125-1130 (2010)

26. Pergolesi, D., Fabbri, E., Cook, S.N., Roddatis, V., Traversa, E., Kilner, J.A.: Tensile lattice distortion does not affect oxygen transport in yttria-stabilized zirconia- $\mathrm{CeO}_{2}$ heterointerfaces. ACS Nano (2012). doi:10.1021/nn302812m

27. Tebano, A., Balestrino, G., Lavanga, S., Martellucci, S., Medaglia, P.G., Paoletti, A., Pasquini, G., Petrocelli, G., Tucciarone, A.: Reflection high-energy electron diffraction oscillations during epitaxial growth of artificially layered films of $\left(\mathrm{BaCuO}_{\mathrm{x}}\right) \mathrm{m} /$ $\left(\mathrm{CaCuO}_{2}\right)$ n. Physica C 355, 335-340 (2001) 